\title{
X-ray Side Bands of Modulated Structures in a $\mathrm{Cu}$-Ti Alloy
}

\author{
By Hiromichi Sagane*, Noriyuki Kuwano** and Kensuke Oki**
}

\begin{abstract}
The side bands of X-ray diffraction were investigated for the modulated structures in a Cu-Ti alloy. The side band profiles in the powder patterns were calculated by using the general diffraction theory for a one-dimensionally disordered crystal. The calculated profiles depend strongly upon the distribution of wavelengths and the wave form of modulation. The side bands are intensified and close to the fundamental reflection for the diffuse distribution of wavelengths. Daniel and Lipson formula is thought to overestimate the mean wavelength of modulation with such a diffuse distribution. The asymmetry in intensity of side bands is mainly caused by the asymmetric wave form of modulation. The experimental X-ray side band profiles were reproduced successfully by the present calculation using proper distributions of the modulation wavelengths and amplitudes. By the comparison of the experimental side band profiles with the calculated ones, it is suggested that the progress in phase separation is accompanied by broadening of the distribution of modulation wavelengths and by increasing in the degree of asymmetry of wave form as well as increasing in the mean wavelength and amplitude.
\end{abstract}

(Received June 19, 1986)

Keywords: $X$-ray side bands, modulated structure, periodic compositional fluctuations, distribution of modulation, wavelength and amplitude, copper-titanium alloy

\section{Introduction}

Some alloys with a modulated structure are known to give side bands in their X-ray diffraction patterns. Since the X-ray side bands of $\mathrm{Cu}-\mathrm{Ni}-\mathrm{Fe}$ alloy were interpreted first by Daniel and Lipson ${ }^{(1)}$ in terms of periodic modulation of composition, a number of models ${ }^{(2)-(6)}$ with an assumed compositional fluctuation mode have been proposed to account for the characteristics of side bands. Simulations of side band profiles were also carried out with various modulation models ${ }^{(7)}$. However, these models could not satisfactorily explain the experimental side band profiles which showed complicated changes associated with the progress in phase separation. The side bands have been used almost only for a rough estimation of the mean wavelength of compositional fluctuations in the modulated structures.

* Department of Metallurgical Engineering, Niihama National College of Technology, Niihama-shi, Ehime 792, Japan.

** Department of Materials Science and Technology, Graduate School of Engineering Sciences, Kyushu University, Kasuga-shi, Fukuoka 816, Japan.
Although the side bands are considered very useful to understand the compositional fluctuations experimentally, they were interpreted hitherto with a single mode of modulation, and no particular attention has been given to the existence of various modes of modulation. It is important, however, to note that there are various wavelengths and amplitudes of modulation coexisting in the actual modulated structures, and they will have a significant influence on the profile of side bands. Electron microscopic studies of the modulated structures in $\mathrm{Cu}-\mathrm{Ti}$ alloys $^{(8)-(11)}$ suggest the coexistence of the modulation with various wavelengths and amplitudes. Tsujimoto et $a l .^{(12)}$ suggested that the diffuse periodicity of modulation contributed to the asymmetry of side band profile. In their interpretation of side bands, a certain function was introduced to describe the diffuse periodicity of modulation. However, in order to understand the side band profile, it is important to investigate in detail the influence of the modulation modes on the side band profile.

In this study, we calculated the side band profiles using the general diffraction theory ${ }^{(13)}$ which took the diffuse periodicity of modula- 
tion into account, and examined the explicit effects of the wavelengths and amplitudes of modulation. The calculated side band profiles were compared with the experimental ones for aged $\mathrm{Cu}-3.7$ at\% $\mathrm{Ti}$ alloys to describe the growth of compositional fluctuations during aging.

\section{Calculation of Side Band Profile}

We consider a crystal structure consisting of $N$ layers, each of which corresponds to one period of the compositional modulation wave. The modulation wave is thought to have a sinusoidal form at an early stage of phase separation and changes its profile gradually to a rectangular form with the development of modulation. Although the profile of modulation wave in actual alloys has no exactly rectangular shape, we assume in this study that the profile is rectangular. The rectangular shape seems to be more reasonable than the sinusoidal one, because the X-ray side bands actually appear at a relatively later stage of phase separation and the modulation wave seems to have developed well into the rectangle-like form by the stage ${ }^{(14)(15)}$. The modulation wave is considered to have $S$ kinds of wavelengths, each of which has $M$ different wave forms, and consequently contains totally $(S \times M)$ kinds of wave profiles. Figure 1 illustrates one of the $(S \times M)$ kinds of wave profiles. The wave form is taken into account by introducing a parameter $\alpha_{m}$. It is symmetric when $\alpha_{m}=0.5$ and becomes asymmetric as $\alpha_{m}$ increases from the value of 0.5 . The dimension of the former half period of the modulation wave is given by $\alpha_{m} Q_{s}$, where $Q_{s}$ is the $s$ th wavelength in terms of the number of $f c c$ cells. The amplitude of the modulation wave is assumed to be proportional to $Q_{s}$ and $|c-\bar{c}|$,

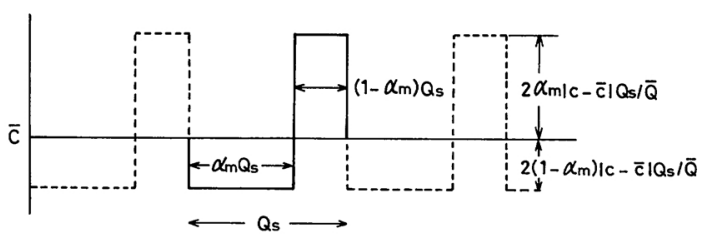

Fig. 1 One of the modulation wave profiles. $\alpha_{m}$ is a parameter describing a wave form. $Q_{s}$ is the wavelength in terms of the number of $f c c$ cells. where $\bar{c}$ is the average composition and $|c-\bar{c}|$ describes the mean amplitude of modulation. The diffraction intensity $I$ from such a structure can be expressed by the general diffraction theory for a one-dimensionally disordered crystal $^{(13)}$,

$$
\begin{aligned}
I= & \left(\overline{V^{2}}+\frac{\overline{V \varphi} \overline{V^{*}}}{1-G}+\frac{\overline{V^{*} \varphi^{*}} \bar{V}}{1-G^{*}}\right) N \\
& +\left\{\overline{V \varphi} \overline{V^{*}} \frac{G^{N}-1}{(1-G)^{2}}+\overline{V^{*} \varphi^{*}} \bar{V} \frac{G^{* N}-1}{\left(1-G^{*}\right)^{2}}\right\}
\end{aligned}
$$

with

$$
\begin{aligned}
& \overline{V^{2}}=\sum_{s=1}^{S} \sum_{m=1}^{M} w_{s} p_{m} V_{s m} V_{s m}^{*}, \\
& \overline{V \varphi}=\sum_{s=1}^{S} \sum_{m=1}^{M} w_{s} p_{m} V_{s m} \exp \left(-2 \pi i H Q_{s} \bar{a}\right), \\
& \bar{V}=\sum_{s=1}^{S} \sum_{m=1}^{M} w_{s} p_{m} V_{s m}
\end{aligned}
$$

and

$$
G=\sum_{s=1}^{S} \sum_{m=1}^{M} w_{s} p_{m} \exp \left(-2 \pi i H Q_{s} \bar{a}\right)
$$

where $w_{s}$ and $p_{m}$ are the existence probabilities for the $s$ th wavelength and the $m$ th wave form, respectively, $V_{s m}$ the structure factor of one period of modulation wave with the $s$ th wavelength and the $m$ th wave form, $H$ the coordinate in the reciprocal lattice for the onedimensionally modulated structure, $\bar{a}$ the average lattice parameter and ${ }^{*}$ means a complex conjugate. The latter half term in eq. (1) can be ignored, because it becomes significant only when $G$ is very close to unity. When $G$ is exactly equal to unity, the intensity $I$ should be

$$
I=N^{2} \bar{V}^{2}+N\left(\overline{V^{2}}-\bar{V}^{2}\right),
$$

but it is replaced by $N \overline{V^{2}}$ in the present calculation. As the modulation occurs along the $\langle 100\rangle$ directions in $\mathrm{Cu}-\mathrm{Ti}$ alloy, the structure factor $V_{s m}$ at $h 00$ in the reciprocal lattice for $f c c$ can be given as follows:

$$
\begin{aligned}
V_{s m}= & \bar{f}\left[\sum_{n=0}^{\alpha_{m} Q_{s}-1}\left\{1-\left(1-\alpha_{m}\right) A_{s}\right\} \exp \left(2 \pi i h L_{n} / Q_{s}\right)\right. \\
& \left.+\sum_{n^{\prime}=0}^{\left(1-\alpha_{m}\right) Q_{s}-1}\left(1+\alpha_{m} A_{s}\right) \exp \left(2 \pi i h L_{n}^{\prime} / Q_{s}\right)\right]
\end{aligned}
$$


with

$$
\begin{array}{r}
L_{n}=n\left\{1-\left(1-\alpha_{m}\right) B_{s}\right\} \\
\text { for } n=0, \cdots,\left(\alpha_{m} Q_{s}-1\right)
\end{array}
$$

and

$$
\begin{aligned}
L_{n}^{\prime}= & \left(\alpha_{m} Q_{s}-1 / 2\right)\left\{1-\left(1-\alpha_{m}\right) B_{s}\right\} \\
& +\left(1+\alpha_{m} B_{s}\right) / 2+n^{\prime}\left(1+\alpha_{m} B_{s}\right) \\
& \text { for } n^{\prime}=0, \cdots,\left\{\left(1-\alpha_{m}\right) Q_{s}-1\right\},
\end{aligned}
$$

where $\bar{f}$ is the average atomic scattering factor, $A_{s}$ the modulation amplitude of scattering factor and $B_{s}$ one of lattice parameters. In the present calculation, the amplitude of the modulation wave is assumed to be proportional to the wavelength $Q_{s}$, as illustrated in Fig. 1. Then $A_{s}$ and $B_{s}$ are given by

$$
A_{s}=2\left\{\left(f_{\mathrm{Ti}}-f_{\mathrm{Cu}}\right) / \bar{f}\right\}|c-\bar{c}|\left(Q_{s} / \bar{Q}\right)
$$

and

$$
B_{s}=2\{(d a / d c) / \bar{a}\}|c-\bar{c}|\left(Q_{s} / \bar{Q}\right)
$$

with

$$
\bar{Q}=\sum_{s=1}^{S} w_{s} Q_{s},
$$

where $f_{\mathrm{Cu}}$ and $f_{\mathrm{Ti}}$ are the atomic scattering factors of the alloy elements, $\mathrm{d} a / \mathrm{d} c$ the derivative of the lattice parameter with respect to the composition. The probabilities $w_{s}$ and $p_{m}$ are assumed to be given by proper binomial distributions. We used the values of $f_{\mathrm{Cu}}=20.778, f_{\mathrm{Ti}}=13.885, \quad \bar{f}=20.524^{(16)}, \mathrm{d} a /$ $\mathrm{d} c=0.00055 \mathrm{~nm} / \mathrm{at} \% \mathrm{Ti}^{(17)}$ and $\bar{a}=0.363 \mathrm{~nm}$. In order to compare directly with the experimental profiles, the calculated intensity is convoluted by a proper Gaussian distribution and integrated in the reciprocal space to convert to powder diffraction. The Lorentzpolarization factor is also taken into account.

\section{Experimental Procedure}

The $\mathrm{Cu}$-Ti alloy used in this study was prepared by induction melting in vacuum. The composition of the alloy was chemically analyzed to be 3.7 at $\% \mathrm{Ti}$. The ingot of the alloy was rolled to sheets about $2 \mathrm{~mm}$ in thickness and homogenized at $1173 \mathrm{~K}$ in vacuum. The sheets were filed into powder finer than $0.1 \mathrm{~mm}$ in size. The powder specimens were sealed in evacuated silica capsules and heated at $1173 \mathrm{~K}$ for $3.6 \mathrm{ks}$ so as to have a state of $\alpha$ phase of $f c c$ structure. Subsequently, they were aged isothermally at 673 or $723 \mathrm{~K}$ for various durations in a salt bath so as to have a modulated structure. The X-ray powder patterns around $h k l=200$ reflection were measured for the aged specimens using a diffractometer with filtered $\mathrm{Cu}-\mathrm{K}_{\alpha}$ radiation. A part of the sheet specimens were further rolled and heat-treated in the same way as for the powder specimens. Transmission electron microscopic observation was carried out for the specimens.

\section{Results and Discussion}

Figure 2 shows the change in profile of the $\mathrm{X}$-ray side bands around 200 reflection for a $\mathrm{Cu}-3.7$ at\% Ti alloy aged at $723 \mathrm{~K}$. The side bands become stronger and closer to the fundamental reflection with the aging time. The high angle side band is more intensified than the low angle one. The difference in intensity between the high and the low angle side band becomes large with increasing aging time. As seen in several studies ${ }^{(8)(10)(18)(19)}$, the mean wavelength of modulation is usually estimated by using Daniel and Lipson formula ${ }^{(1)}$,

$$
Q=\frac{h \tan \theta}{\left(h^{2}+k^{2}+l^{2}\right) \Delta \theta},
$$

where $Q$ is the modulation wavelength in terms of the number of unit cells, $\Delta \theta$ the angular displacement of the position between the side band maximum and the fundamental reflection with $h k l$ and $\theta$ the Bragg angle. However, in case of $\mathrm{Cu}-3.7$ at \% Ti alloy, it is not easy to estimate $\Delta \theta$ accurately, because the side band profile is quite diffuse as seen in Fig. 2. Similar difficulty is found in other alloys. Even when $\Delta \theta$ can be estimated, it is suggested that the formula does not always give a correct value for the mean wavelength of modulation with a diffuse distribution of wavelengths. Figure 3 shows the dependence of the side band profile on the wavelength distribution. When the wavelength distribution becomes diffuse, the side bands are intensified and the maximum 


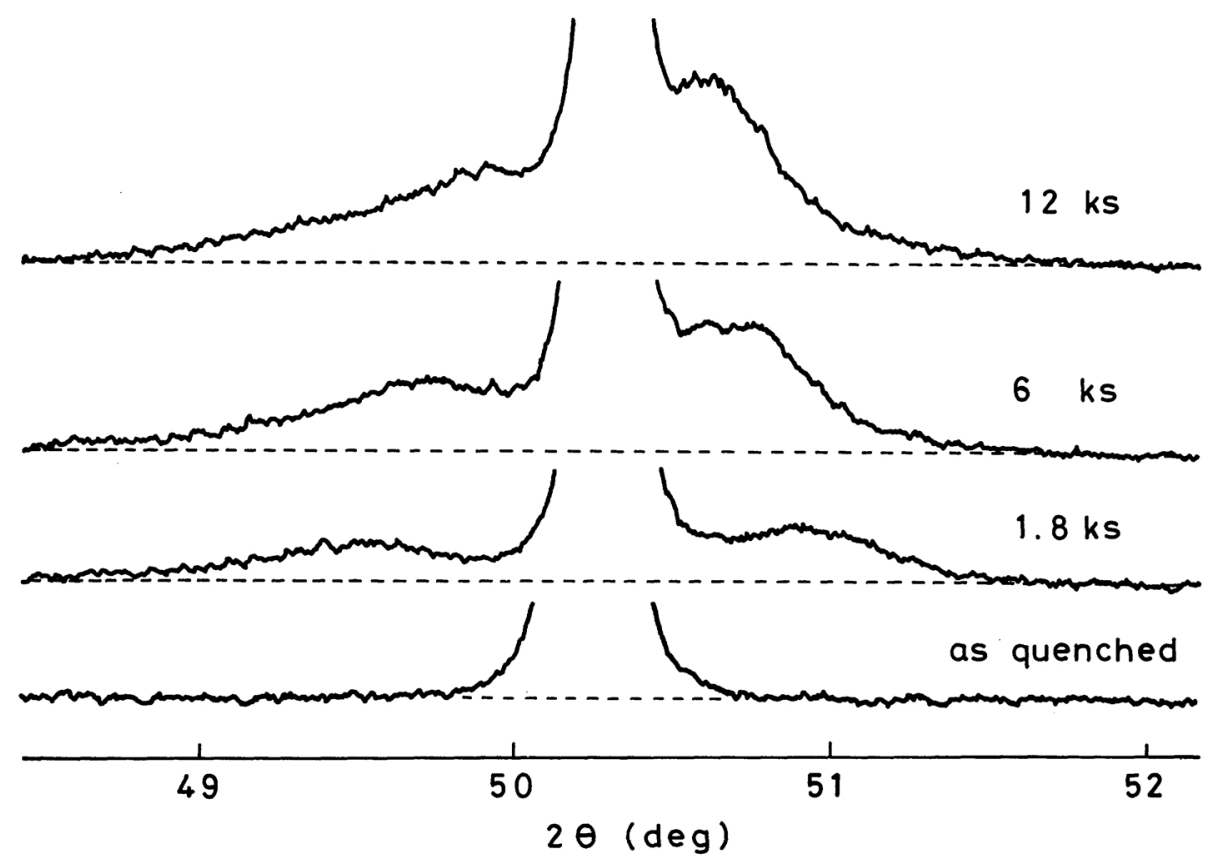

Fig. 2 X-ray side band profiles around 200 reflection for the powder specimens of $\mathrm{Cu}-3.7$ at $\% \mathrm{Ti}$ alloy aged at $723 \mathrm{~K}$.

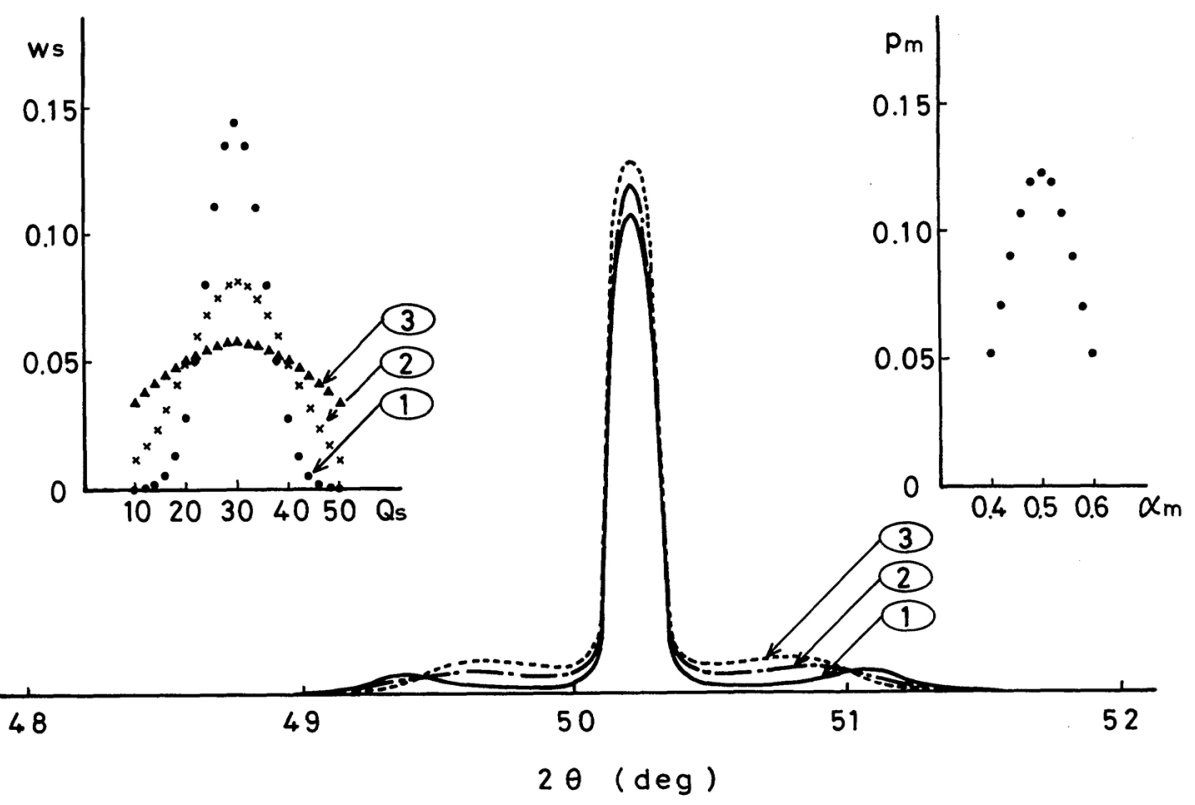

Fig. 3 Dependence of the 200 side band profile on the distribution of wavelengths. The distributions of wavelengths $Q_{s}(s=1-S)$ and the parameters $\alpha_{m}(m=1-M)$ describing the wave forms are shown in the insets. The profiles (1), (2) and (3) are for the wavelength distributions marked with the corresponding number. The side band profiles were calculated with $S=21$, $\bar{Q}=30, M=11, \bar{\alpha}=0.5$ and $|c-\bar{c}|=1.8$ at $\% \mathrm{Ti}$, where $\bar{Q}$ and $\bar{\alpha}$ are the mean values of $Q_{s}$ and $\alpha_{m}$, respectively. 
position becomes close to the fundamental reflection. If Daniel and Lipson formula is applied to the calculated profiles, it leads to $Q=31,38$ and 45 , respectively, while the true value is $\bar{Q}=30$. The deviation from the true value is seen to increase with the diffuseness of the wavelength distribution. It should be pointed out that the side band profile depends strongly upon the wavelength distribution, and the formula gives incorrectly a large value for the mean wavelength in case of the diffuse distribution of wavelengths. Such a diffuse distribution may be actually realized at a later stage of aging. Figure 4 shows the modulated structures in the $\mathrm{Cu}-3.7$ at\% Ti alloy. The morphology exhibits a coexistence of various modulation modes.

It is seen that the calculated side band profiles in Fig. 3 are almost symmetric with respect to the fundamental reflection. But the experimental side band profile at a later stage of aging is usually asymmetric, as shown in Fig. 2.
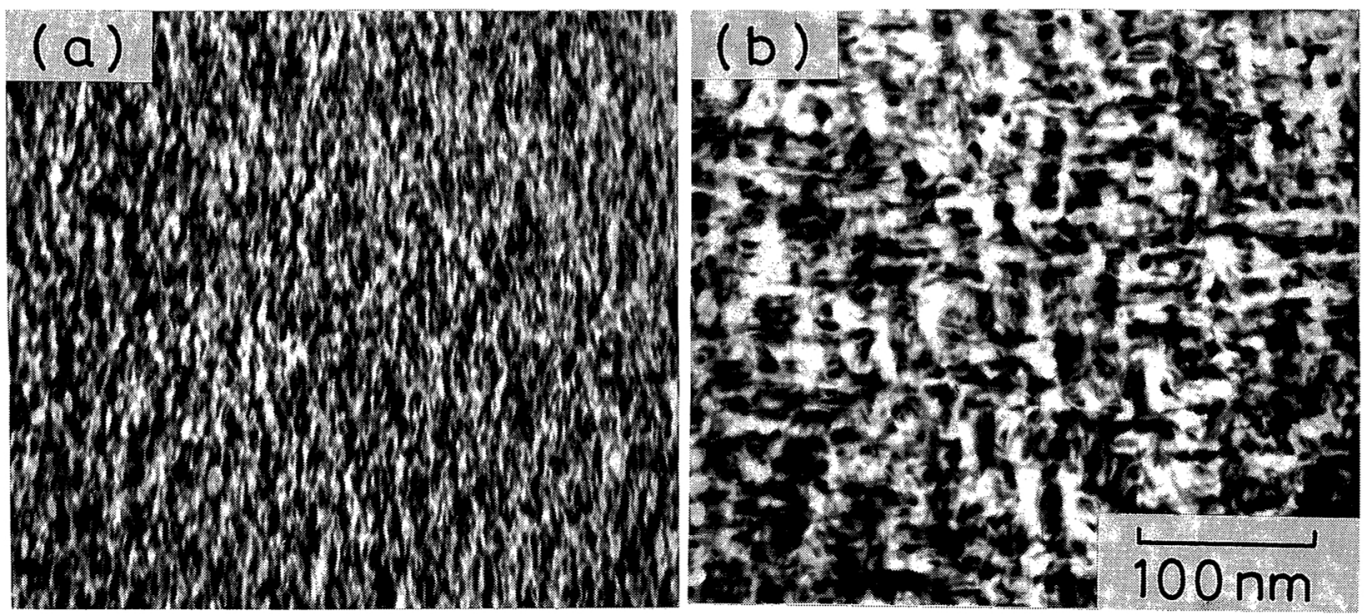

Fig. 4 Electron micrographs of the modulated structures in $\mathrm{Cu}-3.7$ at $\% \mathrm{Ti}$ alloy aged at $673 \mathrm{~K}$ for (a) $3.6 \mathrm{ks}$ and (b) $150 \mathrm{ks}$.

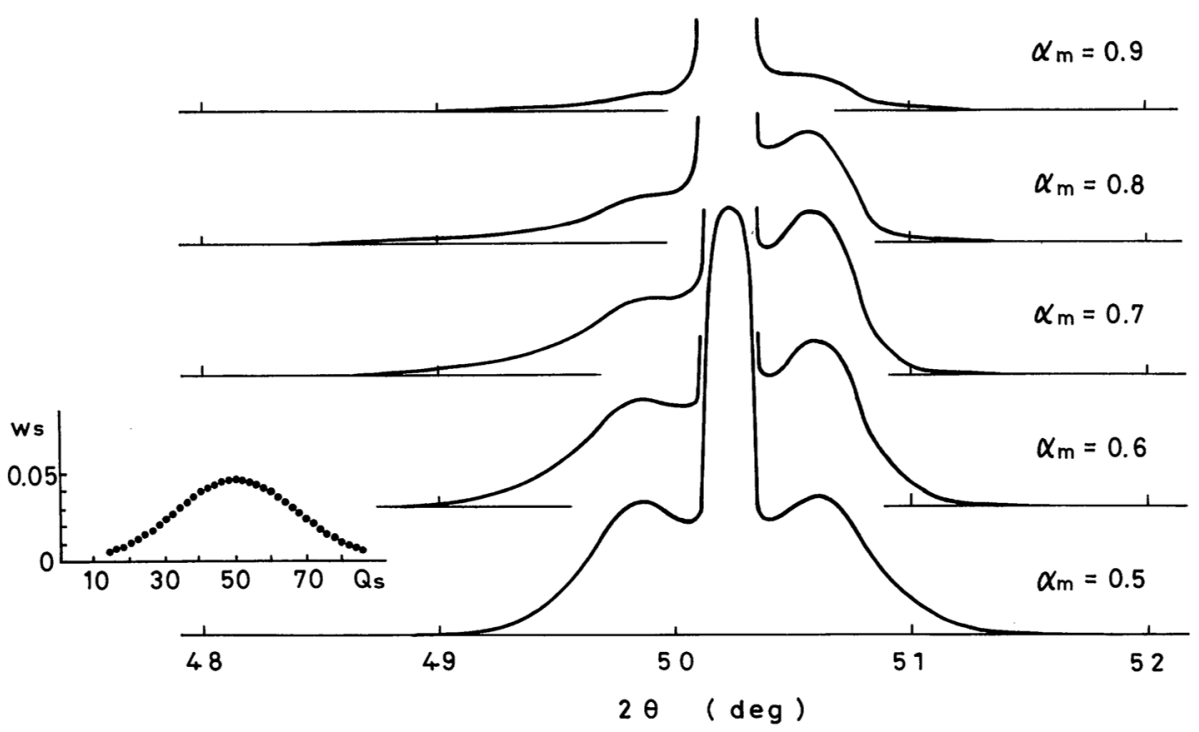

Fig. 5 Dependence of the 200 side band profile on the wave form of the modulation with $S=37$, $\bar{Q}=50, M=1$ and $|c-\bar{c}|=3.8$ at $\% \mathrm{Ti}$. The distribution of $Q_{s}$ is inset in the figure. 


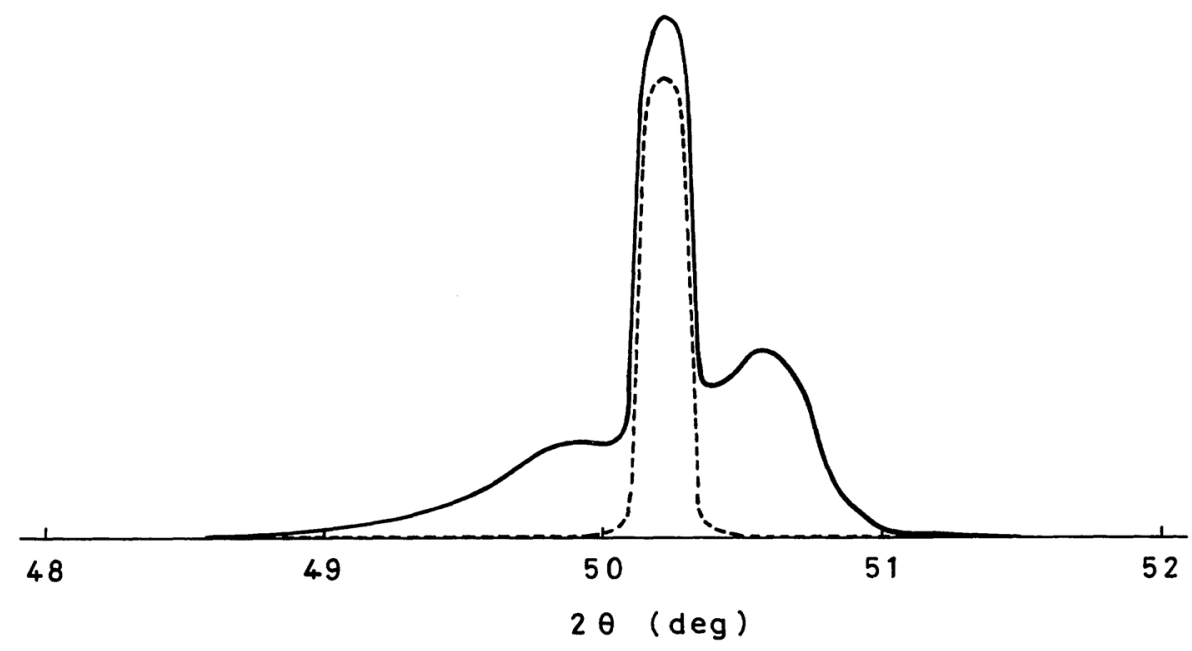

Fig. 6 The 200 side band profiles due to the modulation of lattice parameter (solid line) and atomic scattering factor (broaken line). The profiles are calculated with $S=37, \bar{Q}=50, M=1, \alpha_{m}=0.7$ and $|c-\bar{c}|=3.8$ at $\% \mathrm{Ti}$.

Such an asymmetric profile is reproduced by assuming an asymmetric wave form of modulation. Figure 5 shows the variation of the side band profiles for $\alpha_{m} \geqq 0.5$ with $M=1$. The side band profile is almost symmetric in case of the symmetric wave form with $\alpha_{m}=0.5$, and as $\alpha_{m}$ increases, the side band profile gradually becomes more asymmetric. Figure 6 shows the side band profiles in a fictitious case for either modulation of atomic scattering factor or that of lattice parameter. It is clearly seen that the modulation of atomic scattering factor, or the intensity modulation, has little contribution to the formation of side bands, and the appearance of side bands is due to the modulation of lattice parameter, or the phase modulation. Therefore, the asymmetry of the side band profile can be attributed to the asymmetric wave form of the lattice parameter modulation.

We calculated the side band profiles with appropriate values for $Q_{s}, \alpha_{m},|c-\bar{c}|$ and distributions for $Q_{s}$ and $\alpha_{m}$ so as to reproduce the experimental profiles for various stages of aging. In the calculation, although the distribution of $\alpha_{m}$ seems to change with the progress in phase separation, an invariable broadness is assumed during aging for simplicity. The effect of $\mathrm{K}_{\alpha 2}$ radiation on the side band profile is not considered. Since the experimental side band pro- files for the alloy aged at $723 \mathrm{~K}$ are already shown in Fig. 2, we show below the results for aging at $673 \mathrm{~K}$. Figure 7 (a) shows the experimental side band profile of the alloy aged for $3.6 \mathrm{ks}$. The fundamental reflection is of doublet, the smaller component being due to $\mathrm{K}_{\alpha 2}$ radiation. Figure $7(\mathrm{~b})$ shows the profile calculated with $S=15, \bar{Q}=20, M=11, \bar{\alpha}=0.5$ and $|c-\bar{c}|=1.5$ at $\% \mathrm{Ti}$, where $\bar{\alpha}$ is the mean value of $\alpha_{m}$. Figure $7(\mathrm{c})$ indicates a representative modulation wave form and the distributions of $Q_{s}$ and $\alpha_{m}$. The profile of the calculated side bands is weak and diffuse in agreement with the experimental one. Although it is not easy to specify the modulation wave at this stage of aging, because of the weak side bands, it seems that the modulation has comparatively short wavelengths with a sharp distribution and the wave forms are nearly symmetric. It should be noted that such weak and diffuse side bands are possible to compare with the experimental ones by applying the present calculation. Figure 8(a) shows the experimental side band profile for the subsequent stage of aging. The profile has a tendency that the intensity of high angle side band is stronger than that of low angle. The calculated side band profile for $S=25, \bar{Q}=30$, $M=11, \quad \bar{\alpha}=0.7$ and $|c-\bar{c}|=2.5$ at $\% \mathrm{Ti}$ is shown in Fig. 8(b). Figure 8(c) indicates a repre- 


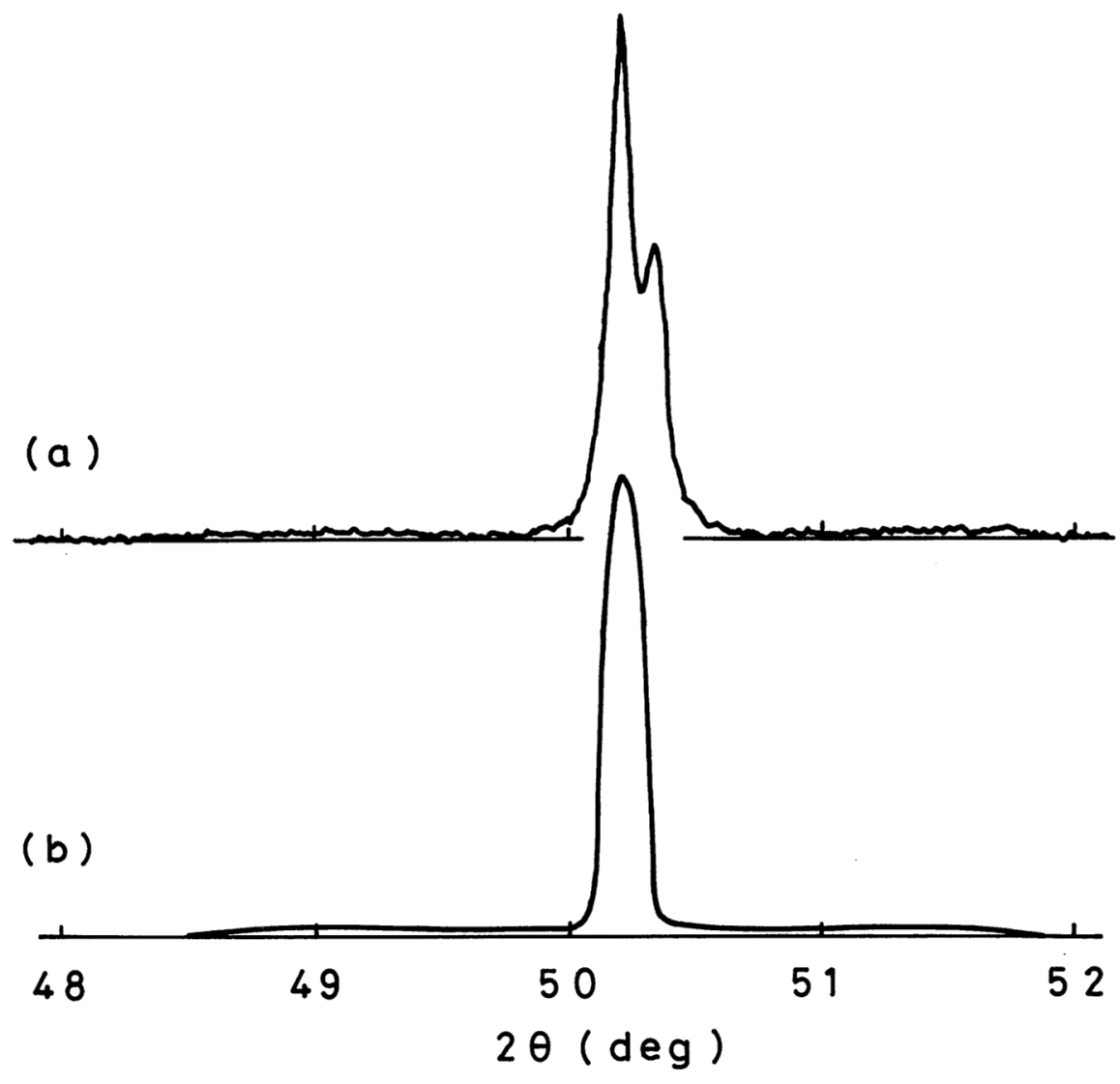

(c)
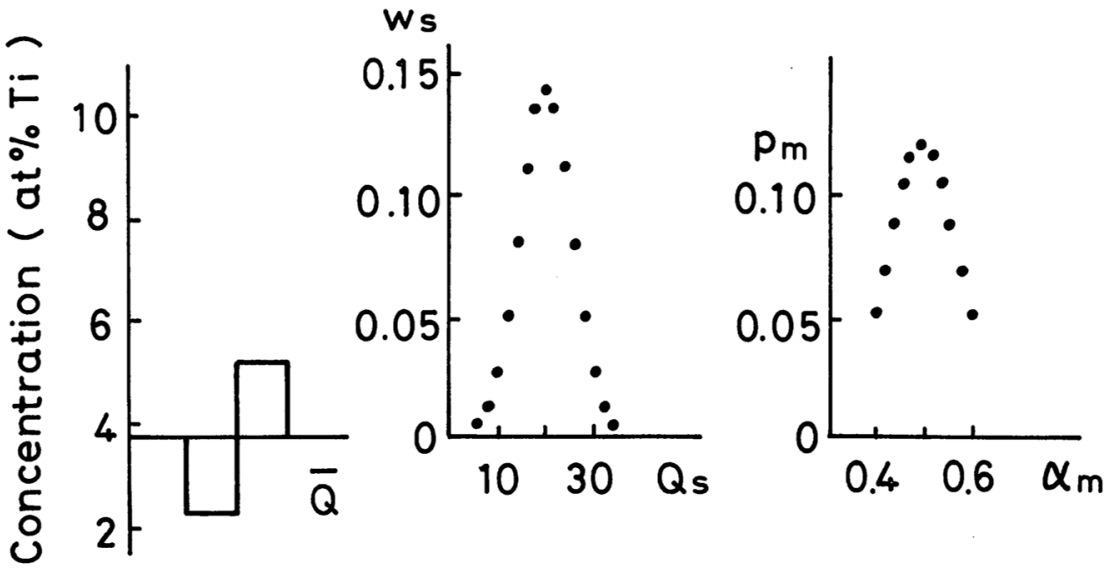

Fig. 7 The 200 side band profiles at an early stage of aging. (a) Experimental result for the alloy aged at $673 \mathrm{~K}$ for $3.6 \mathrm{ks}$, (b) calculated one and (c) representative modulation wave form and the distributions of $Q_{s}$ and $\alpha_{m}$. 


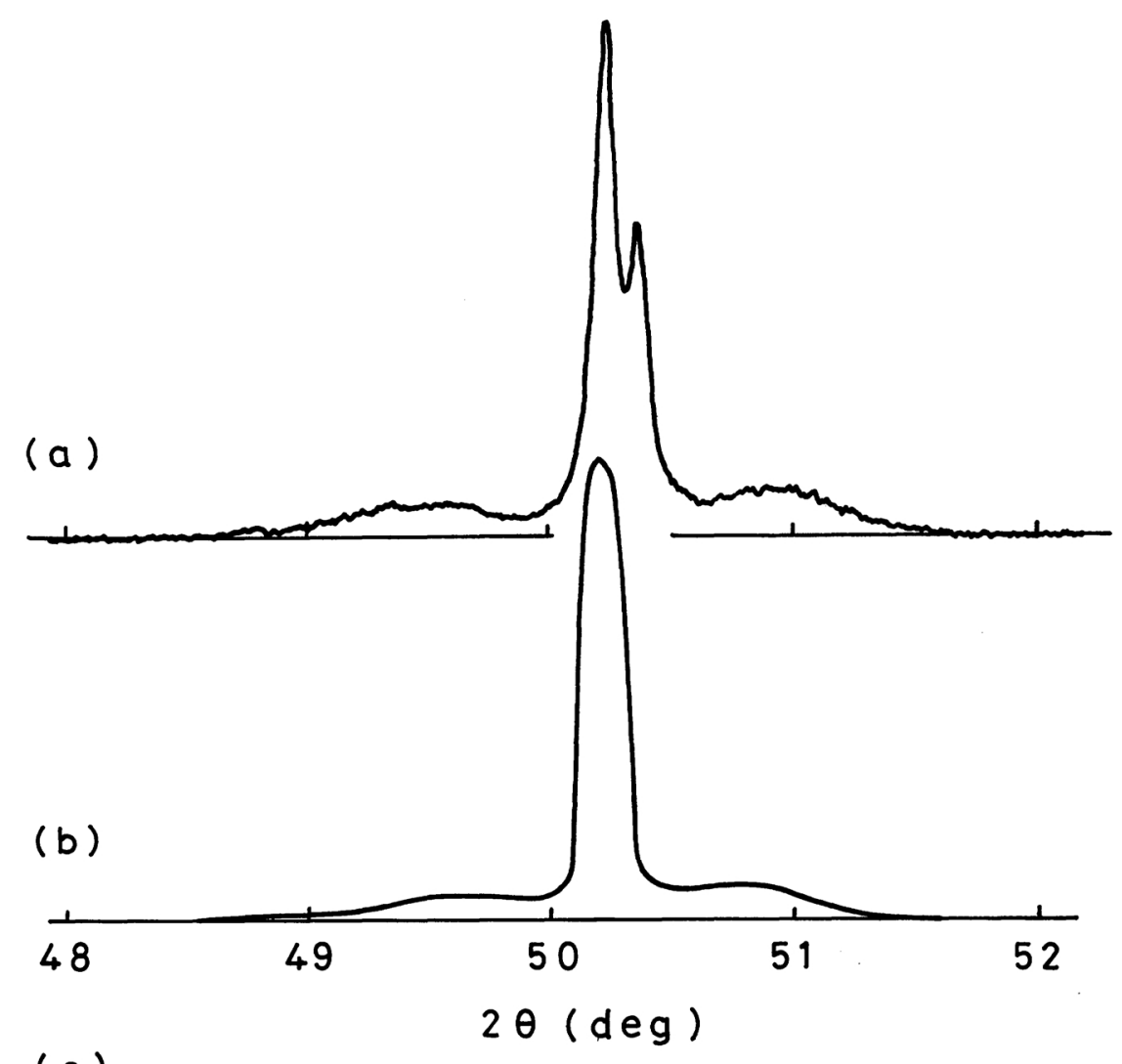

(c)
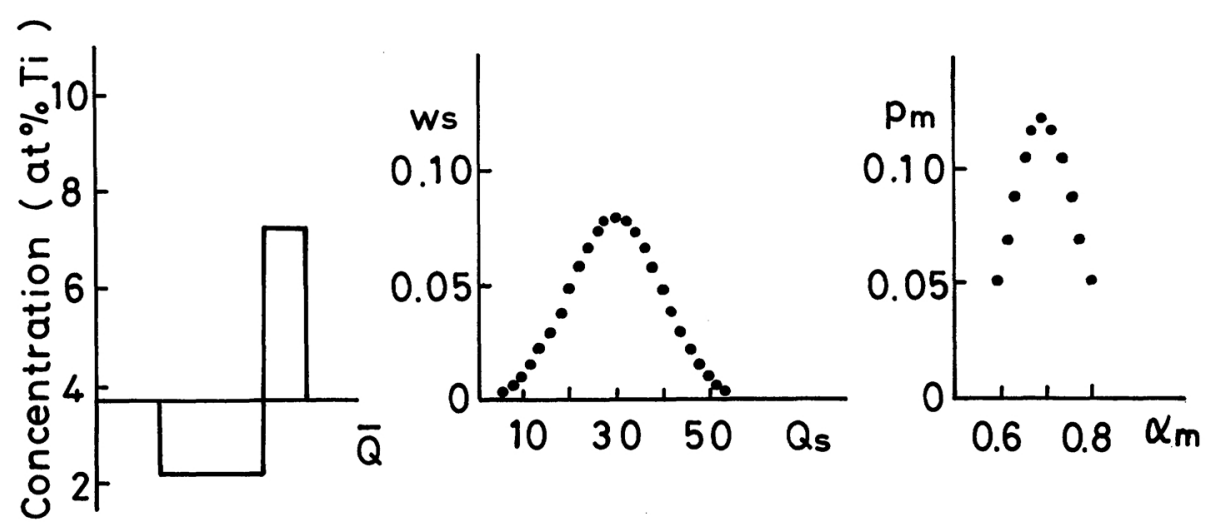

Fig. 8 The 200 side band profiles at the subsequent stage of aging. (a) Experimental result for the alloy aged at $673 \mathrm{~K}$ for $30 \mathrm{ks}$, (b) calculated one and (c) representative modulation wave form and the distributions of $Q_{\mathrm{s}}$ and $\alpha_{\mathrm{m}}$.

sentative modulation wave form and the distributions of $Q_{s}$ and $\alpha_{m}$. The distribution of $Q_{s}$ and the value of $\bar{Q}$ indicate that the modulation has grown to have larger wavelengths and amplitudes with a broader distribution than the modulation in Fig. 7. The value of $\bar{\alpha}=0.7$ denotes that the modulation has asymmetric wave forms as shown in Fig. 8(c). It is seen that the asymmetry of the side band profile is reproduced well in Fig. 8(b). Figures 9(a) and 10(a) 

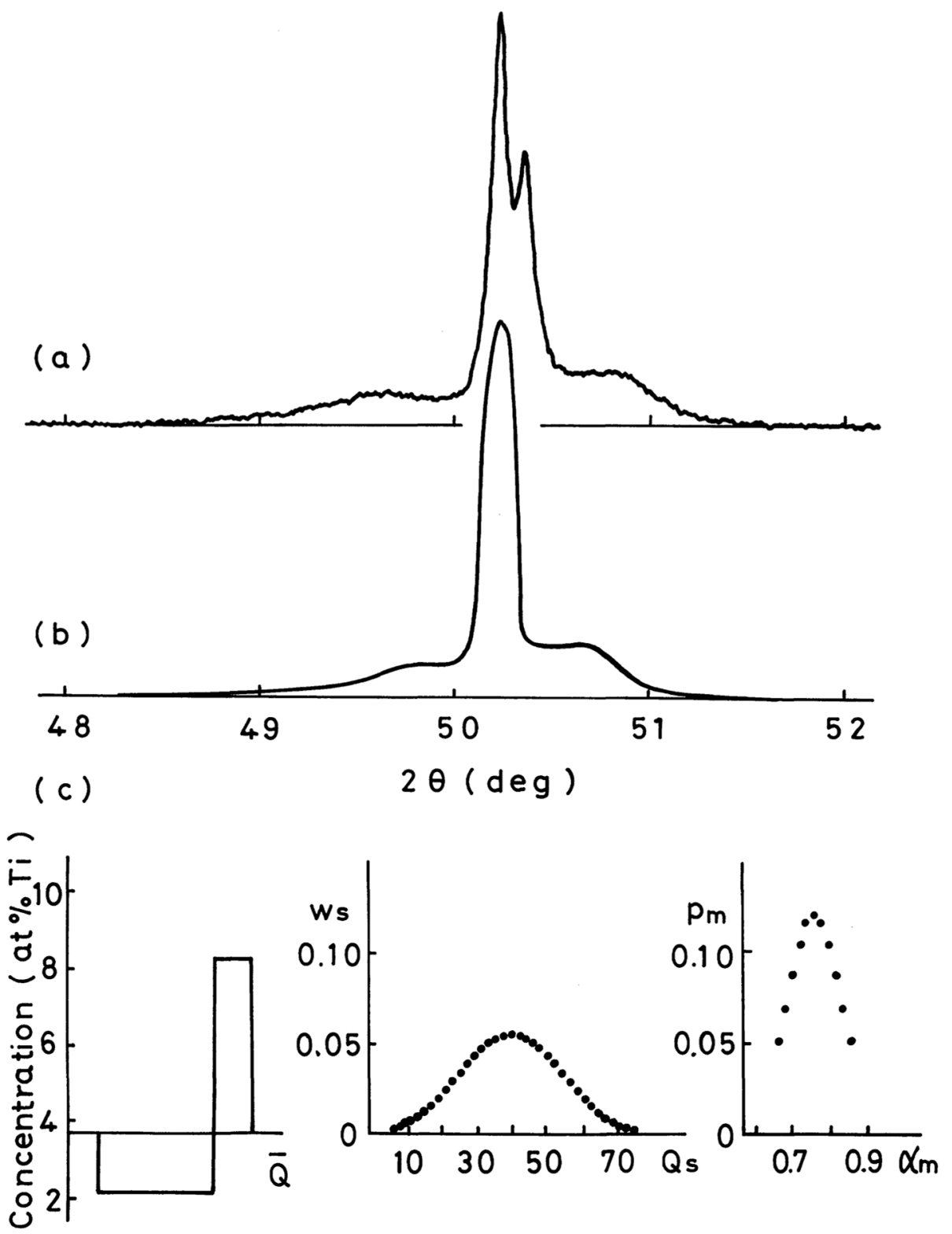

Fig. 9 The 200 side band profiles at the stage of further aging. (a) Experimental result for the alloy aged at $673 \mathrm{~K}$ for $60 \mathrm{ks}$, (b) calculated one and (c) representative modulation wave form and the distributions of $Q_{\mathrm{s}}$ and $\alpha_{\mathrm{m}}$.

show the side band profiles for the alloys further aged. As the modulation develops, the side bands are more intensified, more asymmetric and closer to the fundamental reflection. The corresponding side band profile calculated for $S=35, \bar{Q}=40, M=11, \bar{\alpha}=0.75$ and $|c-\bar{c}|=3$ at $\% \mathrm{Ti}$ is shown in Fig. 9(b), and the one for $S=37, \bar{Q}=50, M=11, \bar{\alpha}=0.8$ and $|c-\bar{c}|=3.8$ at $\% \mathrm{Ti}$ in Fig. 10(b). The calculated profiles denote that the modulation is growing further with increasing wavelength and with increasing degree of asymmetry of wave form. If Daniel and Lipson formula is applied to the side bands in Fig. 10(a), it gives 

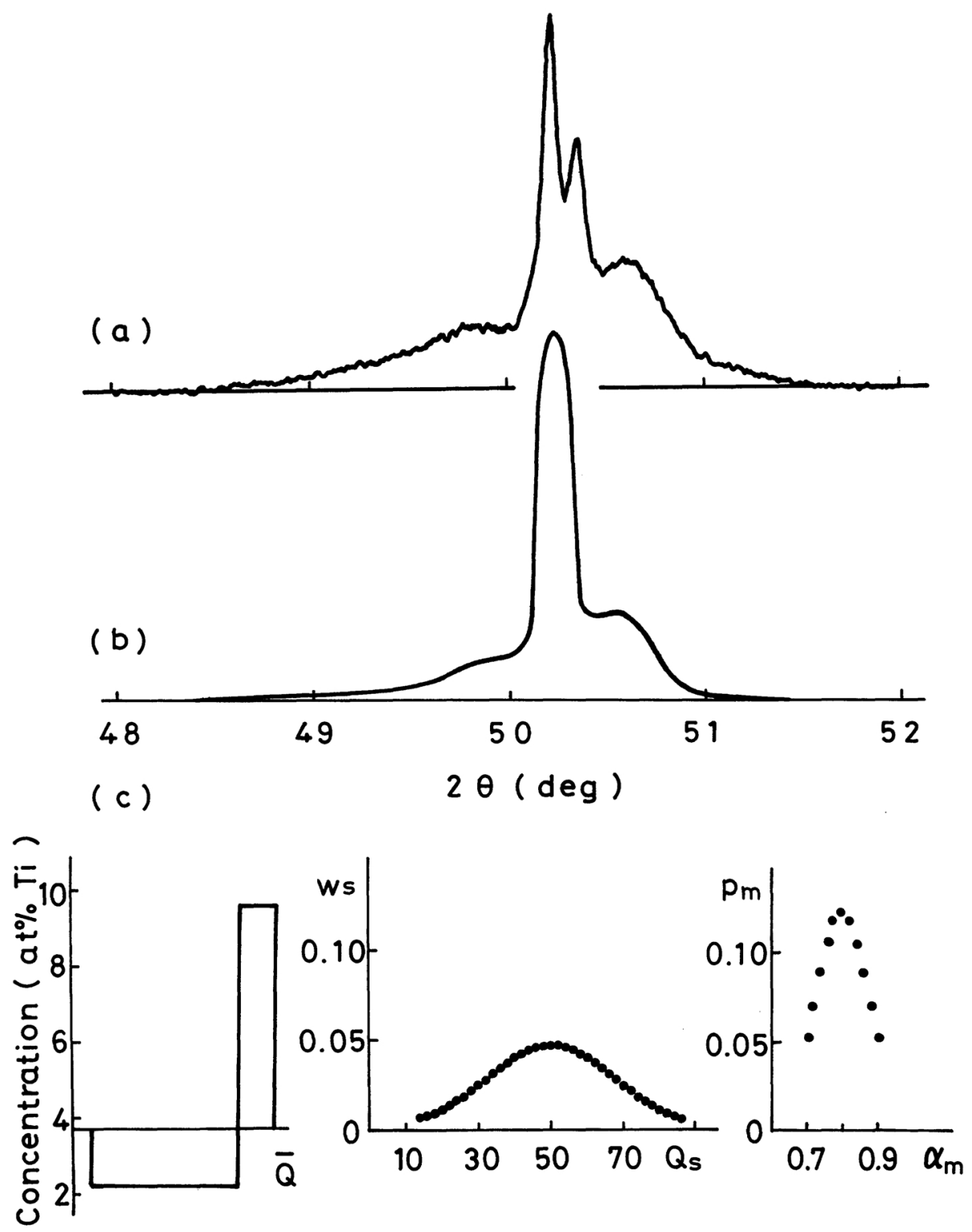

Fig. 10 The 200 side band profiles at the later stage of aging. (a) Experimental result for the alloy aged at $673 \mathrm{~K}$ for $180 \mathrm{ks}$, (b) calculated one and (c) representative modulation wave form and the distributions of $Q_{s}$ and $\alpha_{m}$.

$\bar{Q}=71$, while Fig. $10(\mathrm{~b})$ is calculated with $\bar{Q}=50$. If the diffuse distribution of wavelengths is realized in $\mathrm{Cu}-\mathrm{Ti}$ alloy, the formula is found again to give a larger value for the mean wavelength. Attention has been directed only to the position of the maximum intensity in order to apply the formula. But it is necessary to give attention to the whole profile for the interpretation of the side band profile.

The changes in side band profile observed strongly suggest that in the process of aging in the alloy, the modulation arises from small compositional fluctuations with a sharp distribution of wavelengths and the nearly symmetric wave form, and grows to large fluctuations with a broad distribution of wavelengths 

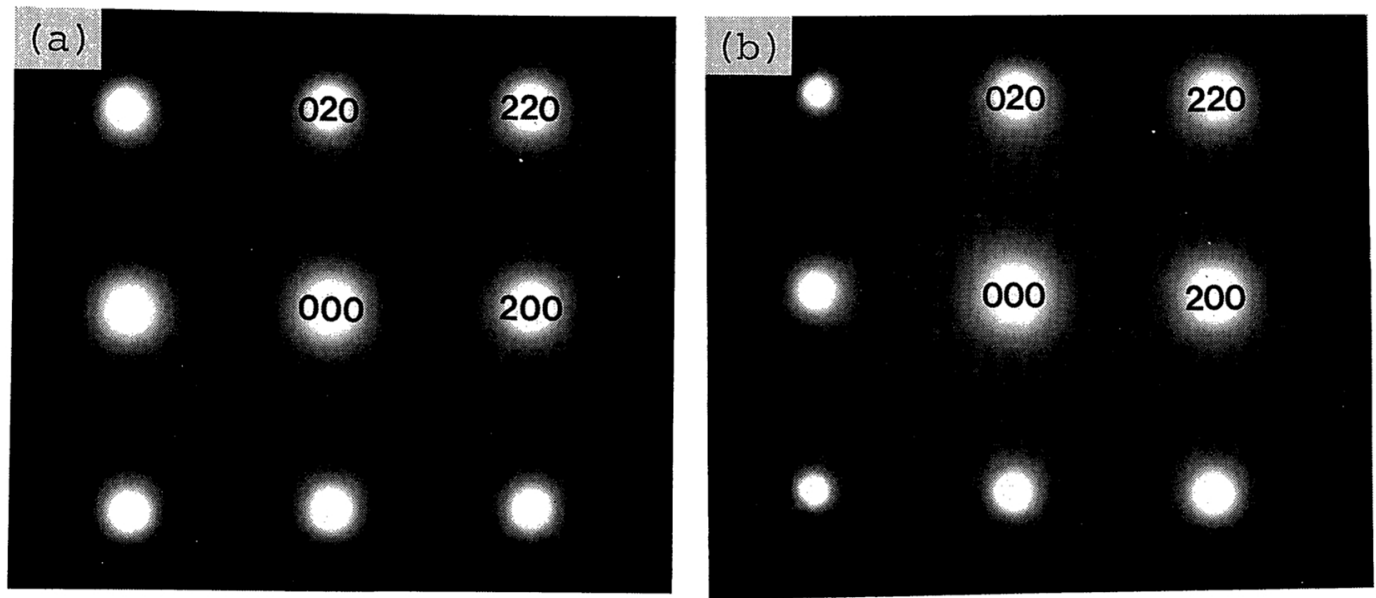

Fig. 11 Electron diffraction patterns with [001] incidence for the alloys aged at $673 \mathrm{~K}$ for (a) $3.6 \mathrm{ks}$ and (b) $180 \mathrm{ks}$

and the strongly asymmetric wave forms. The asymmetry of wave form seems to be caused by the phase separation in the low $\mathrm{Ti}$ region of $3.7 \mathrm{at} \% \mathrm{Ti}$, where the clusters of a high concentration of $\mathrm{Ti}$ are expected to be formed periodically with a small fraction. The present analysis of the side band profiles suggests, as shown in Figs. 7 to 10 , that the mean amplitude and wavelength of modulation increase simultaneously. In a spinodal decomposition, the mean amplitude is known to be almost constant at a later stage where the mean wavelength increases ${ }^{(15)(20)}$, in disagreement with the present result. In this alloy of 3.7 at $\%$ $\mathrm{Ti}$, however, the behavior of the modulation may be in a complicated state which is caused by the phase separation near the spinodal line ${ }^{(21)}$ and the formation of the metastable $\beta^{\prime}$ phase with the $D 1_{a}$ superlattice structure of $\mathrm{Cu}_{4} \mathrm{Ti}^{(10)}$. The formation of the $\beta^{\prime}$ phase is considered to be related with the enrichment of $\mathrm{Ti}$ in the modulation and stimulated in the higher $\mathrm{Ti}$ regions with increasing mean amplitude of modulation. In order to examine the formation of the $\beta^{\prime}$ phase, electron diffraction patterns were observed for the alloys aged at 673 $\mathrm{K}$ for $3.6 \mathrm{ks}$ and for $180 \mathrm{ks}$, the alloys corresponding to those shown in Figs. 7 and 10, respectively. As seen in Fig. 11, the alloy gives no superlattice reflections after $3.6 \mathrm{ks}$ but shows the distinct ones after $180 \mathrm{ks}$, indicating the formation of the $\beta^{\prime}$ phase during the aging. The in- crease in the mean amplitude of modulation during the aging is suggested experimentally by the formation of the $\beta^{\prime}$ phase.

\section{Conclusion}

The compositional fluctuations in the modulated structures of aged $\mathrm{Cu}-\mathrm{Ti}$ alloys have been investigated by means of X-ray powder diffraction. The experimental side band profiles were reproduced by the general diffraction theory, and they were found to be explained well by taking account of the distribution of wavelengths and the asymmetric wave forms of modulation. The side bands were characterized by the following results.

(1) The side bands are attributed to the phase modulation of the periodic change in lattice parameter.

(2) The side bands are intensified and close to the fundamental reflection by the diffuse distribution of wavelengths.

(3) The asymmetry of the side band profile is mainly caused by the asymmetric wave form of modulation.

(4) On the process of aging, the compositional fluctuations grow with broadening of the wavelength distribution and with increasing degree of asymmetry of wave form as well as increasing mean wavelength and amplitude of modulation. 


\section{Acknowledgment}

This work was supported partially by a Grant-in-Aid for Scientific Research given by the Ministry of Education, Science and Culture of Japan.

\section{REFERENCES}

(1) V. Daniel and H. Lipson: Proc. Roy. Soc., A181 (1943), 368.

(2) M. E. Hargreaves: Acta Cryst., 4 (1951), 301.

(3) T. J. Tiedema, J. Bouman and W. G. Burgers: Acta Met., 5 (1957), 310.

(4) E. Biedermann: Acta Cryst., 13 (1960), 650.

(5) A. Guinier: Acta Met., 3 (1955), 510.

(6) M. Hillert, M. Cohen and B. L. Averbach: Acta Met., 9 (1961), 536.

(7) D. de Fontaine: Local Atomic Arrangements Studied by X-ray Diffraction (Ed. by J. B. Cohen and J. E. Hilliard), Gorden \& Breach, New York, (1966), p. 51.

(8) J. A. Cornie, A. Datta and W. A. Soffa: Met. Trans., 4 (1973), 727.
(9) D. E. Laughlin and J. W. Cahn: Acta Met., 23 (1975), 329.

(10) A. Datta and W. A. Soffa: Acta Met., 24 (1976), 987.

(11) J. Dutkiewicz: Met. Technol., 5 (1978), 333.

(12) T. Tsujimoto, K. Hashimoto and K. Saito: Acta Met., 25 (1977), 295.

(13) J. Kakinoki and Y. Komura: J. Phys. Soc. Japan, 7 (1952), 30.

(14) T. Tsujimoto: Trans. JIM, 22 (1981), 127.

(15) T. Miyazaki, T. Kozakai, S. Mizuno and M. Doi: Trans. JIM, 24 (1983), 246.

(16) P. A. Doyle and P. S. Turnur: Acta Cryst., A24 (1968), 390.

(17) S. Saji, S. Ikeno and S. Hori: J. Japan Inst. Metals, 42 (1978), 63 (in Japanese).

(18) T. Miyazaki, E. Yajima and H. Suga: Trans. JIM, 12 (1971), 119.

(19) T. Tsujimoto and K. Saito: J. Japan Inst. Metals, 37 (1973), 173 (in Japanese).

(20) T. Miyazaki: Bulletin Japan Inst. Metals, 15 (1976), 309 (in Japanese).

(21) T. Tsujimoto: J. Japan Inst. Metals, 40 (1976), 521 (in Japanese). 